\title{
Sciences de l'éducation, raison pédagogique et rationalité économique: faut-il vraiment diaboliser la réforme HarmoS?
}

\section{Maurice Tardif}

Ce texte est une réaction critique aux thèses du professeur Herzog à propos des risques de standardisation de l'école et d'instrumentalisation des sciences de l'éducation que comporterait, selon lui, la réforme HarmoS. Je soutiens que le professeur Herzog propose un critique beaucoup trop radicale de la pensée instrumentale, de l'économie et de l'approche par standards en éducation. La scolarisation moderne est depuis toujours une réalité profondément économique et instrumentale: nier cette réalité conduit selon moi à adopter une vision naïve de l'éducation et des standards, tout en s'interdisant la possibilité de rationaliser les pratiques et institutions éducatives. Enfin, je tâcherai de montrer que le professeur Herzog surestime le potentiel critique des sciences de l'éducation.

Comme universitaire nord-américain qui travaille aujourd'hui en Suisse (et qui a eu aussi l'occasion d'œuvrer dans différents pays), on m'a invité à réagir au texte que le professeur Walter Herzog a écrit à propos des risques de standardisation de l'école et d'instrumentalisation des sciences de l'éducation que comporterait, selon lui, la réforme HarmoS.

Essentiellement, si j'ai bien compris le cœur de l'argumentation du professeur Herzog, ces risques seraient liés à la mise en œuvre, au sein du monde scolaire et de la profession enseignante, d'une logique de standardisation issue du monde économique. Cette logique des standards, dont la norme ISO constituerait en quelque sorte le paradigme international, aurait pour conséquence de défigurer les réalités éducatives (autant les réalités institutionnelles que professionnelles et interindividuelles), de les réduire à une vision strictement mécanique, productiviste et quantitative, tout en affectant en profondeur le potentiel critique et théorique des sciences de l'éducation qui seraient, dans tout ce processus, subordonnées à la politique selon une conception étroitement instrumentale et empiriste de la recherche éducative.

De manière générale, en lisant et relisant ce texte, j'éprouve le sentiment d'être devant ce que j'appellerai une critique exacerbée et unilatérale de la pensée instrumentale, de l'économie et de l'approche par standards en éducation. Je n'ai 
aucun doute que cette impression de radicalisme que donne le texte découle des raccourcis critiques auxquels a dû se livrer le professeur Herzog pour exposer des idées complexes en si peu de pages. La pensée critique en sciences sociales et humaines a ordinairement besoin d'espace pour s'exprimer pleinement avec toutes les nuances nécessaires. Lorsque le texte est trop court, comme c'est le cas ici, la critique doit alors forcément éliminer les nuances et travailler pour ainsi dire davantage à la hache qu'au scalpel!

Dans les pages qui suivent, je me propose de reprendre certaines des principales thèses du professeur Herzog afin de mettre en lumière leur radicalisme beaucoup trop unilatéral. Chemin faisant, je vais introduire un certain nombre d'idées et d'arguments qui permettront de voir sous d'autres angles, dont quelques-uns peut-être plus positifs, la réforme HarmoS et la logique des standards.

Thèse 1: La réforme HarmoS et la mise en place d'un pilotage de l'éducation conçu comme système sont avant tout une tentative politique d'introduction de la logique économique, voire industrielle en éducation. La standardisation est un processus d'asservissement de l'éducation et des sciences de l'éducation à l'économie.

\begin{tabular}{|c|c|}
\hline L'éducation (positif) & Léconomie (négatif) \\
\hline Réalités qualitatives, significations, subjectivités & Phénomènes quantitatifs, mesures, contrôles \\
\hline Monde humain & Environnement technologique \\
\hline Complexité, mouvance, multidimensionnalité & Mécanique, cybernétique, technique \\
\hline Pensée théorique critique & Objectivisme axiologiquement neutre \\
\hline Contenus, processus, interactions, contextes & Compétences, outputs, standards, abstractions \\
\hline
\end{tabular}

Cette première thèse sous-tend tout le texte du professeur Herzog et lui donne tout son sens. J'ai le sentiment que cette thèse présente l'éducation et les sciences de l'éducation comme des vierges effarouchées qui ne veulent surtout pas entretenir de relations charnelles avec l'économie, elle-même conçue de manière strictement négative. Bref, nous sommes en présence d'une relation quasi bipolaire entre l'éducation et l'économie. Cette bipolarité est clairement normative, car elle indexe constamment l'économie de valeurs négatives et l'éducation de valeurs positives. Elle structure toute l'argumentation du professeur Herzog et elle est constamment reprise dans divers doublets conceptuels qui organisent le texte à travers un système d'oppositions de ce genre:

Comme je ne peux me lancer dans une longue argumentation, je vais énoncer très brièvement quelques idées qui n'entrent pas dans le système bipolaire du professeur Herzog, mais dont il devrait, me semble-t-il, tenir compte dans son argumentation.

- L'éducation et plus particulièrement la scolarisation sont depuis toujours des réalités profondément économiques. En vérité, l'industrialisation et la scola- 
risation constituent, avec l'émergence des sociétés modernes, deux processus sociaux étroitement imbriqués et inconcevables l'un sans l'autre. À partir du $19^{\mathrm{e}}$ siècle, la forme scolaire sera pour une large part une reprise et une adaptation progressive de la forme industrielle (et j'ajoute: militaire) à l'éducation: centralisation des décisions, planification systémique, traitement de masse des élèves, uniformisation des pratiques scolaires, formalisation d'un ordre règlementaire, etc. Cela est encore plus vrai dans les sociétés actuelles où le système productif exige un système éducatif capable de produire et reproduire sans arrêt une main-d'œuvre hautement qualifiée et compétente. Bref, le monde économique dans lequel nous vivons est impensable sans l'école et l'université actuelles. En réalité, les sciences elles-mêmes font désormais partie intégrante des forces productives.

- De manière plus prosaïque, en éducation, je crois qu'il faut cesser de jouer les vierges offensées dès qu'on parle d'économie et de rationalisation économique. C'est un fait que l'éducation actuelle coûte extrêmement cher aux états nationaux et donc aux populations qui payent taxes et impôts. Je rappelle que depuis les années 1950, les gouvernements des sociétés occidentales ont investi massivement en éducation et consacrent en moyenne, selon les décennies, entre $20 \%$ et $30 \%$ de leurs budgets à leurs systèmes éducatifs. Or, près de $75 \%$ de cet investissement public va en salaires pour les agents scolaires. Dans ce cadre, il me semble parfaitement normal que ceux-ci aient un jour ou l'autre des comptes à rendre à la «rationalité économique» et surtout aux pouvoirs élus, ainsi qu'aux citoyens qui les financent parfois très grassement. Le temps est fini (a-t-il même existé?) où on pouvait dépenser sans compter. Bien sûr, je ne crois pas que tout se ramène en éducation à des choix économiques; toutefois, n'en déplaise à certains pédagogues de la gauche-caviar, je considère que ceux-ci sont aussi centraux que les questions pédagogiques ou culturelles: il faut donc cesser de les évacuer. Je suis en train d'écrire ce texte alors que je suis dans une région pauvre du Brésil (une petite ville près de Curitiba dans l'état du Paraná) où je suis invité à prononcer une série de conférences. Alors, un peu méchamment, j’ai envie de dire que seuls des gens très instruits et très bien payés, comme certains professeurs des universités suisses et canadiennes, peuvent croire qu'ils n'ont pas de comptes économiques à rendre et que personne n'a le droit d'essayer de mesurer leur performance académique ou les retombées concrètes de leurs recherches.

- En ce qui concerne l'asservissement des sciences de l'éducation à l'économie, je ne sais trop ce que cette thèse veut dire au juste. Les sciences de l'éducation sont apparues dans la foulée de l'édification des grands systèmes scolaires au $19^{\mathrm{e}}$ siècle et surtout au $20^{\mathrm{e}}$ siècle, et en raison de la nécessité qui en découlait d'introduire des mesures de contrôles à grande échelle de ces nouvelles institutions et pratiques socio-éducatives. Elles ont été constamment mobilisées pour garantir l'amélioration des pratiques et des systèmes scolaires, et la formation de leurs agents. Dès leur origine, les sciences de l'éducation ont été 
largement régies par une rationalité instrumentale au service des pouvoirs étatiques qui cherchaient à mettre de l'ordre dans une sphère publique en pleine expansion. Les sciences de l'éducation s'inscrivent donc dans le projet de gouvernance rationnelle (ou de domination si on préfere une lecture à la Foucault) du social propre à la modernité. Encore de nos jours, cette logique me semble toujours à l'œuvre, ce qui explique qu'une bonne part des sciences de l'éducation correspond à des discours normatifs dont la fonction principale consiste à «normer» les pratiques et conceptions des agents scolaires, à gérer et impulser des réformes, à dire aux enseignants comment ils devraient enseigner pour "agir en véritables professionnels», à s'immiscer dans les processus d'apprentissages scolaires pour les orienter selon les dernières théories à la mode comme, par exemple, le socioconstructivisme ou la méthode globale de lecture.

Au final, il découle de tout ceci que, contrairement à ce que pourrait laisser croire les propos du professeur Herzog, tous nos systèmes éducatifs ainsi que les sciences de l'éducation sont largement et depuis fort longtemps pétris de rationalité économique et instrumentale. Que les rapports complexes entre l'économie et l'éducation changent aujourd'hui de sens et d'ampleur est une thèse sur laquelle on peut discuter, mais prétendre que leur introduction dans le champ éducatif intervient avec la réforme HarmoS me semble donc une fiction historique ou rhétorique.

\section{Thèse 2: La réforme HarmoS conduit à ignorer la fonction critique du travail} scientifique et le caractère théoriquement construit et controversé des faits scientifiques.

La vision épurée et noble proposée par le professeur Herzog à propos des sciences de l'éducation et de la recherche éducative me semble faire l'impasse sur plusieurs problèmes.

- Le professeur Herzog semble adhérer à une conception strictement poppérienne de l'activité scientifique, laquelle monte forcément en épingle la fonction critique et argumentative de la science et accentue fortement le rôle moteur des controverses dans le progrès de la connaissance. Pourtant, s'il est exact que la critique et les controverses sont importantes en science, elles ne forment pas pour autant le cœur de l'activité scientifique normale (la «science normale» de Thomas Kuhn), laquelle consiste pour l'essentiel à produire des théories cohérentes et stables capables de rendre compte d'une large moisson de faits largement reconnus par la communauté scientifique. Les scientifiques actuels ne sont pas des philosophes athéniens: leur objectif n'est pas d'engendrer des controverses et des disputes argumentatives, mais d'accroître leurs connaissances et de les fortifier en s'appuyant sur des faits les mieux établis possible. Bien sûr, un "fait» devient un "fait scientifique» une fois qu'il a été reconnu tel dans le cadre d'une théorie scientifique, laquelle le «filtre» à tra- 
vers des critiques et des arguments; mais une fois cette reconnaissance réalisée, le fait en question fourni une base solide pour faire progresser la connaissance et favoriser la découverte d'autres faits qui lui sont liés. En ce sens, s'il est exact que tous les "faits» sont toujours imprégnés de théories, il n'en reste pas moins que certaines théories proposent une lecture de certains «faits» qui rallie, à une époque donnée, l'ensemble de la communauté scientifique, et ce parfois pendant plusieurs décennies, voire plusieurs siècles. De ce point de vue, mettre l'accent exclusivement sur la fonction critique et controversée des faits scientifiques laisse croire que tous les faits et théories scientifiques sont toujours en train d'être contestés et critiqués, ce qui est faux. En réalité, dans toutes les sciences, les scientifiques s'appuient dans leur travail quotidien sur des faits admis (et j'ajoute: des théories bien établies) par tous leurs collègues, et c'est à partir d'eux qu'ils travaillent et font progresser leur discipline. Or, c'est sans doute là que le bât blesse vraiment pour les sciences de l'éducation.

- En effet, à mon avis, le principal problème des sciences de l'éducation, problème que ne semble pas voir le professeur Herzog, réside, non pas dans les menaces virtuelles qui pèseraient sur leur fonction critique, mais dans la faiblesse généralisée et l'éclatement global de leurs théories, ainsi que dans la pauvreté scientifique des faits sur lesquels elles s'appuient bien souvent. Je conçois ici les sciences de l'éducation au sens nord-américain du terme, lequel terme englobe donc les sciences sociales et humaines qui ont pour objet l'éducation (sociologie de l'éducation, histoire de l'éducation, psychologie de l'éducation, etc.), les didactiques, la psychopédagogie, l'administration scolaire et divers types de savoirs souvent inclassables: les théories de la gestion de classe, les théories de la motivation, l'orthopédagogie, l'andragogie, l'éducation physique, la philosophie de l'éducation, etc. Il faut comprendre que toutes ces désignations se conjuguent en vérité au pluriel! Par exemple, la sociologie de l'éducation correspond en réalité à une bonne douzaine de sociologies de l'éducation qui n'utilisent pas les mêmes notions, méthodes et théories de référence. Il en va de même de la psychologie de l'éducation: béhaviorisme, constructivisme, socioconstructivisme, psychologie sociale, cognitivisme, humanisme, phénoménologie, psychanalyse, socioanalyse, psychologie expérimentale, etc. Et chacune de ces conceptions se subdivise ellemême en sous-conceptions qui ne partagent pas les mêmes prémisses épistémologiques, scientifiques et méthodologiques, comme par exemple dans le cas du cognitivisme actuel: cognitivisme computationnel, cognitivisme connexionniste, cognitivisme neuronal, cognitivisme néopiagétien, cognitivisme situé, cognitivisme culturel, cognitivisme social, etc. Je n'ose même pas parler ici des nombreuses variantes et sous-variantes continentales, nationales, culturelles et linguistiques que connaissent toutes ces conceptions disparates.

En définitive, je ne crois pas que les sciences de l'éducation doivent s'aplatir devant les pouvoirs politiques ou économiques et renier leur potentiel critique. Ce- 
pendant, si elles veulent éviter de s'aplatir et vraiment faire valoir ce potentiel, elles doivent pouvoir contribuer à la connaissance des faits éducatifs et à l'amélioration des institutions et des pratiques éducatives. Ce qui menace aujourd'hui les sciences de l'éducation, ce n'est pas tant leur subordination à une politique instrumentale que leur incapacité à jouer leur rôle scientifique important, à proposer des théories solides et des faits probants. C'est de là qui vient leur véritable faiblesse et leur risque de subordination à n'importe quelles idéologies et politiques éducatives.

Thèse 3: La pédagogie et l'éducation en général sont irréductibles à une vision techniciste ou mécanique. Elles renvoient à des réalités complexes, à des environnements sociaux et humains: il en découle que "le déficit technologique de la pédagogie est insurmontable».

À cause de sa trop grande généralité, cette thèse ne veut pas dire grand-chose, et surtout, elle ne nous aide pas à mieux comprendre comment, à partir de connaissances, décisions et démarches rationnelles, nous pouvons améliorer nos pratiques et institutions éducatives.

- En effet, tout se passe comme si «le déficit technologique de la pédagogie» interdisait la recherche de causes et d'effets propres aux pratiques éducatives ainsi que la mise en place d'une rationalisation "moyens-fins» de ces mêmes pratiques. Je suis d'accord avec le professeur Herzog: la pédagogie n'est pas une technologie; cependant, dans le cadre de notre société (et de la tradition éducative occidentale), la pédagogie est une activité rationnelle et, à ce titre, on peut s'efforcer de mieux la connaître et de mieux la réaliser. En désignant la pédagogie comme activité rationnelle, j'entends ici une activité orientée par des motifs explicites débouchant sur des buts et qui incorpore un savoir servant de principe régulateur de sa propre réalisation, notamment en termes de coordination des moyens et des buts. Ce savoir incorporé à l'action peut être exprimé en termes d'assertions ou de propositions susceptibles d'être critiquées. Autrement dit, comme toute action rationnelle, l'agir pédagogique est susceptible d'évaluation et donc de contestations, de critiques, etc. Par exemple, des enseignants qui utilisent la méthode globale (whole langage) pour faire apprendre la lecture à de jeunes enfants du primaire utilisent-ils oui ou non une démarche pédagogique conforme au but visé selon l'état des connaissances actuelles? Face à des conflits entre ses élèves, la démarche de résolution préconisée par cette enseignante est-elle valable ou non? Plusieurs élèves ne comprennent pas la matière présentée par cet enseignant, malgré le bon vouloir de celui-ci: sa méthode de présentation est-elle ou non adéquate? Un enseignant doit-il ou non maintenir un contact visuel avec ses élèves pendant une leçon? La supervision de classe de cet enseignant novice est-elle adéquate, permet-elle aux élèves de se centrer sur la tâche? Comment, à quels moments et selon quelle fréquence un enseignant doit-il interroger ses élèves 
du $1^{\text {er }}$ cycle du secondaire dans le cadre d'une leçon de deux heures sur l'histoire de la Suisse? Ces questions ne sont pas technologiques et nous n'aurons jamais de technologies pour les résoudre. Cependant, selon l'état de nos connaissances actuelles des processus d'enseignement et d'apprentissage, nous pouvons espérer apporter des réponses pertinentes à chacune d'elles. Ces réponses, fondées sur des recherches empiriques, peuvent être incorporées à l'action pédagogique, la guider, voire l'améliorer dans certains cas. Confrontées aux pratiques scolaires réelles, ces réponses peuvent être par la suite testées, critiquées, révisées et, par conséquent, améliorées. En ce sens, reconnaître le déficit technologique de la pédagogie et sa complexité communicationnelle ne signifie d'aucune manière traiter la pédagogie comme une sorte d'activité chaotique sur laquelle nous n'aurions aucune prise rationnelle. Si les sciences de l'éducation ne peuvent pas contribuer à l'amélioration des pratiques pédagogiques et à leur connaissance rationnelle, alors il faut fermer les facultés des sciences de l'éducation partout dans le monde.

Thèse 4: Les standards sont des instruments de normalisation qui mesurent des compétences, ce Mantra des réformateurs néolibéraux de la formation. Les standards sont la monnaie d'un discours politique qui cherche des réponses économiques à des questions pédagogiques.

Cette dernière thèse est l'argument principal du texte, c'est-à-dire ce qu'il vise à démontrer. Encore là, il me semble que nous nageons en plein Western sociologique avec des bons éducateurs pédagogues et des méchants néolibéraux réformateurs!

En réalité, je vois mal comment on peut piloter un système éducatif, peu importe l'idéologie politique en présence, sans user de standards normatifs généraux. Certes, de tels standards ont pour inconvénient un haut degré d'abstraction et ils ne portent que sur des résultats quantifiables. Cependant, la régulation à vaste échelle d'un système éducatif peut difficilement faire autrement sans retomber dans quelque chose de pire: une bureaucratisation outrancière des institutions et des pratiques éducatives qui tente de gérer par le bas en imposant tous azimuts des règlementations et des contrôles infinis. L'introduction de standards en éducation (mais aussi dans les domaines de la santé, des services sociaux, etc.) se situe dans l'émergence, depuis les années 80 , de modèles de gestion post-fordiste et post-bureaucratique. Dans une perspective post-fordiste, il s'agit essentiellement non plus de contrôler les contenus et les processus du système scolaire en fixant à l'avance des règles bureaucratiques, mais de fixer des exigences externes (des attentes politiques normatives: des standards) destinées à réguler les résultats (ou outputs). Qui fixe ces exigences? Dans une société démocratique comme la Suisse (ou le Canada), il appartient au pouvoir politique élu et à l'autorité éducative légale de les définir et de les imposer. De tels standards sont forcément des constructions politiques, des attentes sociales de haut niveau de gé- 
néralité; il est donc normal qu'ils soient des objets de débats, de controverses, de luttes idéologiques. Leurs sens, contenus et fonctions peuvent par conséquent évoluer selon les conjonctures politiques, les rapports sociaux de force, les courants d'idées qui traversent la société. Cependant, d'une manière ou d'une autre, l'approche par standards, qui n'a rien de parfait, m’apparaît plus intéressante qu'une approche bureaucratique de l'éducation. Davantage qu'une gestion bureaucratique traditionnelle, elle offre un cadre de travail plus souple pour l'action, tout en fixant des contraintes collectives à l'ensemble des acteurs et institutions éducatives.

Enfin, le professeur Herzog soutient que les compétences sont le Mantra des réformateurs néolibéraux. N'est-ce pas aller encore une fois beaucoup trop vite en affaire, en disqualifiant d'un seul coup des milliers de travaux sur les compétences et des milliers d'expériences éducatives basées sur des approches par compétences? Je ne suis pas un grand défenseur des approches par compétences en éducation. Cependant, je crois qu'il faut leur reconnaître un mérite important: elles cherchent toutes à mettre l'accent sur la transférabilité des apprentissages et des savoirs scolaires dans diverses autres situations scolaires, mais aussi non scolaires. Les approches par compétences ne sont pas toutes issues de l'industrie et de l'économie néolibérale. Elles découlent aussi de la nécessité critique de repenser la formation scolaire autrement qu'en référence aux savoirs scolaires canoniques (grammaire, mathématiques, histoire, géographie, philosophie, etc.). En Europe et en Amérique du Nord, le développement des systèmes scolaires s'est réalisé, entre les années 1950 et 1970, sans vraiment remettre en question les curricula traditionnels basés sur des savoirs académiques et les découpages anciens par matière scolaire. Or, à partir des années 1980, ces curricula semblent de plus en plus inadaptés aux exigences de formation de la société et de l'économie contemporaines d'une part, aux importantes différences entre élèves qui proviennent de toutes les couches de la société d'autre part. Les curricula traditionnels, fondés sur les savoirs scolaires et la culture académique, ne profitent qu'aux héritiers de la culture. C'est pourquoi on observe dans un grand nombre de pays, depuis les années 1990, une série d'importantes réformes des programmes et des formations scolaires, qui mettent l'accent sur les compétences, c'est-à-dire sur une appropriation reconstructive et opératoire des savoirs scolaires afin de les rendre transférables par les élèves dans les diverses situations de la vie courante. Dans cette perspective, plusieurs pédagogues, y compris de gauche, ont repris à leur compte cette perspective qui recoupe, par bien des côtés, les idéaux pédagogiques de l'éducation nouvelle: faire une école ouverte sur la vie, concevoir des apprentissages qui ont un sens et une utilité dans les situations quotidiennes. Bref, tout ceci pour dire qu'on peut bien sûr critiquer les approches par compétences, mais les assimiler sans autre à un Mantra néolibéral me semble une véritable absurdité.

Mots clés: éducation, rationalisation, instrumentalisation, scolarisation, critique 